\title{
Probing the Nature of Ultra-Steep Spectrum Radio Sources
}

\section{Mehreen Mahmud}

JIVE, Oude Hoogeveensedijk 4, 7991 PD Dwingeloo, the Netherlands

E-mail: mahmudejive.nl

\section{Zsolt Paragi}

${ }^{1}$ JIVE, Oude Hoogeveensedijk 4, 7991 PD Dwingeloo, the Netherlands

${ }^{2}$ MTA Research Group for Physical Geodesy and Geodynamics, Penc, Hungary

E-mail: zparagiejive.nl

\section{Huub Röttgering}

Leiden Observatory, Universiteit Leiden, Huygens Laboratory / J.H. Oort Building, Niels Bohrweg 2, NL-2333 CA Leiden, the Netherlands

E-mail: rottgeringestrw. leidenuniv.nl

\section{Hans-Rainer Klöckner}

University of Oxford, Denys Wilkinson Building, Keble Road, Oxford OX1 3RH, UK \& MPIfR, Auf dem Hügel 69, 53121 Bonn, Germany

E-mail: hrkeastro.ox.ac.uk

\section{George Miley}

Leiden Observatory, Universiteit Leiden, Huygens Laboratory/ J.H. Oort Building,

Niels Bohrweg 2, NL-2333 CA Leiden, the Netherlands

E-mail: mileyestrw.leidenuniv.nl

Here we present, first results from e-VLBI observations at $18 \mathrm{~cm}$ on a small sample of ultra-steep spectrum sources (spectral index between $74 \mathrm{MHz}$ and $325 \mathrm{MHz}<-1.4$ ), that are unresolved at the arcsecond level, in an attempt to elucidate the nature of these (as yet) poorly understood sources. A number of possible scenarios have been previously proposed for the origin of these sources which include: radio galaxies located near or at the epoch of reionization, young obscured radio galaxies, steep spectrum core quasars and pulsars. Presented here are first detections on milliarcsecond scales (positions, flux densities and radio morphologies) of two of these sources, $0722+291$ and $1512+470$, that are a first step in understanding their radio morphologies and classification. These results show that a moderate fraction of ultra-steep spectrum sources may have compact structures that can be studied only at the highest resolution with the VLBI technique.

10th European VLBI Network Symposium and EVN Users Meeting: VLBI and the new generation of radio arrays

September 20-24, 2010

Manchester UK 


\section{Introduction}

Radio sources with an ultra steep radio spectrum (USS) (defined here as having $\alpha<-1.4$, $S_{v} \propto v^{\alpha}$ ) are often associated with extreme phenomenon in the Universe. USS sources with a classical FRII type radio morphology are for a large part associated with very distant galaxies $2<z<5.2$ [4][8]. Being very massive and often located in proto-clusters, these USS sources can be used to study the origin and evolution of massive galaxies and clusters. Recent results include (i) the finding that massive radio galaxies with masses of around $10^{12} \mathrm{M}_{\odot}$ exists up to $z \sim 5$ [9] and (ii) the discovery of proto-clusters up to $z \sim 4$ with total masses as expected for progenitors of nearby clusters (a few times $10^{14} \mathrm{M}_{\odot}$ [11]). USS sources with mostly diffuse radio emission are virtually always associated with clusters up to $z \sim 0.5$ [10]. The emitting regions can have spectacular sizes of up to a few Mpc. The most likely explanation is that the radio emitting plasma traces shocks in merging clusters.

The study of these two types of USS sources both started in the late seventies with studies of their radio morphology. Follow-up work with large optical and X-ray telescopes subsequently gave an understanding of their importance for studies of galaxy and cluster evolution. A third class of USS objects has not yet received much attention. These are the ones that are unresolved on arcsecond scales. The aim of these observations is to set a first step towards elucidating the nature of these objects. There are a number of possible scenarios for the origin of these objects, including: (i) radio galaxies located near or at the epoch of reionization (as suggested by the well known spectral index redshift correlation [5]), (ii) young obscured radio galaxies, with the early phase accompanied by a starburst perhaps ([7] inferred star formation rates of $\sim 1000$ solar masses per year), (iii) steep spectrum core quasars and (iv) pulsars.

\section{Sample Selection}

To obtain the sample, we correlated the $74 \mathrm{MHz}$ VLSS catalog [1] with the WENSS $325 \mathrm{MHz}$ catalog [6] and selected $0.02 \%$ of about $10^{5}$ VLSS sources with the required ultra steep spectrum. To obtain sources that are compact on arcsecond scales, we then selected sources that (i) were unresolved by the FIRST $1400 \mathrm{MHz}$ survey [3] and (ii) had a flux ratio between the $1400 \mathrm{MHz}$ NVSS [2] and FIRST catalog in the range 0.8 to 1.2. The latter criteria ensures that FIRST survey has captured all the flux from the sources and therefore reliably can measure that the emission comes from scales less then about 5 arcsecond. The FIRST and NVSS maps were also visually inspected to ensure that the structures of the sources were compact and unresolved, and that there were no nearby sources that could have led to ambiguities in the measured fluxes in the sample.

\section{Observations}

The data for five USS sources was obtained at L-band (18cm) on 10 and 11 June 2010 using the EVN in e-VLBI mode, in a ten hour run on both days. The scheduled data rate was $1024 \mathrm{Mb} / \mathrm{s}$. Presented in this paper are initial results for two of the sources, $0722+291$ and $1512+470$. Both sources were phase-referenced to close calibrators, J0736+2954 and J1500+4751 respectively. The flux densities, spectral indices (based on the 75 and $325 \mathrm{MHz}$ fluxes) and the flux ratios between 
Table 1: Integrated flux densities (mJy): 0722+291 \& 1512+470

\begin{tabular}{lcccccccc}
\hline Target & $\alpha^{\dagger}$ & Ratio $^{\ddagger}$ & $S_{V L S S}$ & $S_{\text {WENSS }}$ & $S_{\text {FIRST }}$ & $S_{N V S S}$ & $S_{V L B I}^{2}$ & $\%^{\mp}$ \\
\hline $0722+291$ & -1.44 & 1.09 & 650 & 76 & 10 & 9 & 1.9 & 27 \\
$1512+470$ & -1.49 & 0.98 & 1130 & 123 & 217 & 220 & 42 & 25 \\
\hline
\end{tabular}

${ }^{\dagger}$ Spectral indices, calculated from the WENSS [6] and VLSS [1] survey flux densities

Ratio of the NVSS [2] and FIRST [3] flux density measurements

'Flux density measurements from our 1.66GHz e-VLBI observations

${ }^{\mp}$ Estimated fraction of recovered flux density at $1.66 \mathrm{GHz}$
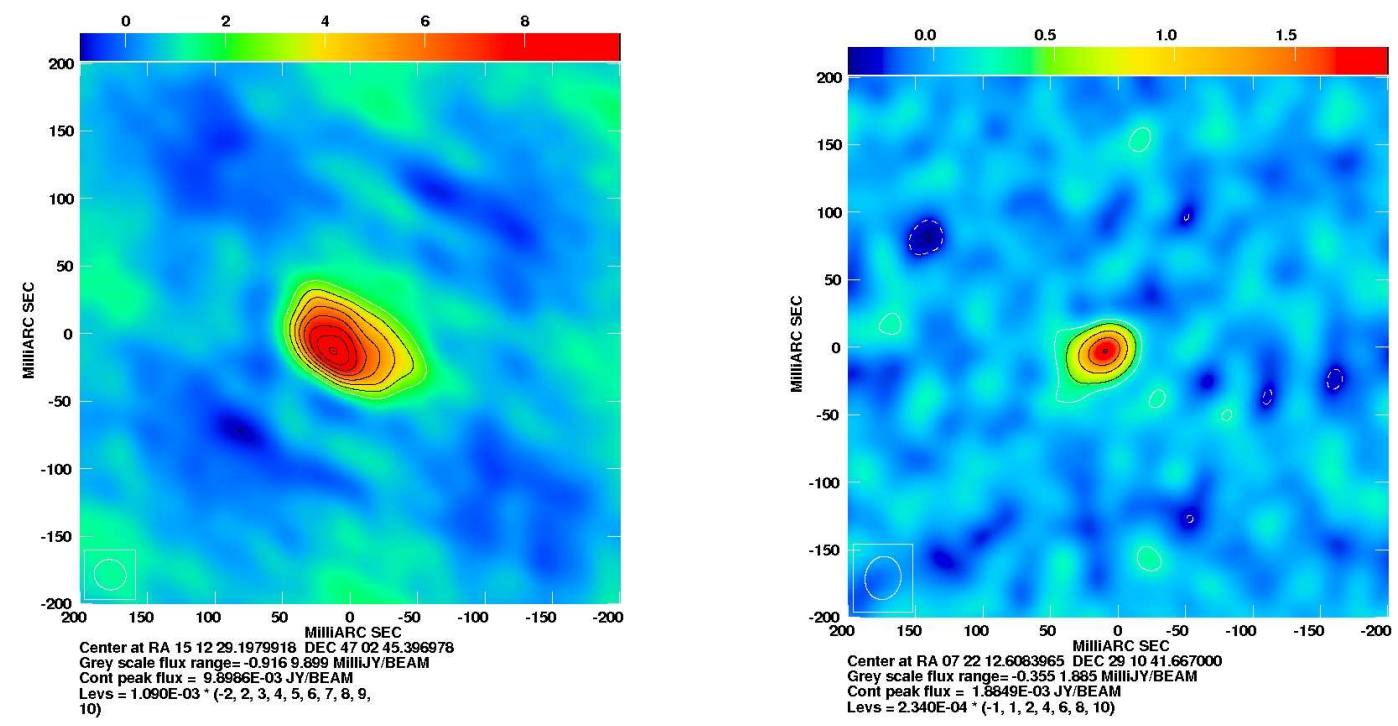

Figure 1: L-Band EVN total intensity maps of $1512+470$ (left) and $0722+291$ (right). The color bar on top shows the Flux distribution in millijansky. For $1512+470$, the peak $I$ contour is $9.9 \mathrm{mJy} / \mathrm{Beam}$, the $\mathrm{rms}$ is $\sim 0.36 \mathrm{mJy}$ and the restoring beam is $23.88 \times 22.31$ mas at a position angle of $70.07^{\circ}$. For $0722+291$, peak $I$ contour is $1.88 \mathrm{mJy} / \mathrm{Beam}$, the rms is $\sim 0.085 \mathrm{mJy}$ and the restoring beam is $32.15 \times 26.43$ mas at a position angle of $-9.95^{\circ}$.

FIRST and NVSS surveys for two sources presented here, are listed in Table 1. Also presented in the table are our flux density measurements from e-VLBI observations.

\section{Results}

Our first results show two detections of our target sources from an initial sample of five sources: $0722+291$ and $1512+470$ with total flux densities of $\sim 2 \mathrm{mJy}$ and $42 \mathrm{mJy}$, respectively. $0722+291$ does not show clear evidence of structure on VLBI scales and is consistent with a point source model, albeit at significantly lower flux density than detected by the VLA. 1512+470 on the other hand is clearly resolved with a peak brightness to total flux density ratio of 1:4. Total intensity contour maps are shown for both sources in Figure 1. We obtained lower limits for the brightness temperatures of $1512+470$ as $5.1(1+z) \times 10^{6} \mathrm{~K}$, and of $0722+291$ as $1.5(1+z) \times 10^{6} \mathrm{~K}$. The visibilities in the amplitude versus UV-distance plot show evidence of extended structure, but these 
cannot be constrained well because of the limited $u v$-coverage in our short snapshot observations, and lack of short spacings.

\section{Conclusion}

Because there are no redshifts available, it is difficult to classify these sources. But from our initial observations we can currently make some tentative conclusions. The fluxes of 1512+470 from Table 1 indicate that the spectrum probably consists of a steep component between 74 and $325 \mathrm{MHz}$ and a self-absorbed core component that could be indicative of a restarting AGN. We note however that the structural appearance and low observed brightness temperature makes this interpretation unlikely. In case of $0722+291$ we cannot exclude the AGN origin because the detected source component is very compact and thus $T_{\mathrm{b}}$ may be very significantly underestimated. The associated emission could also possibly be from a pulsar in the center of a nebula. We also note that while both sources have compact or somewhat resolved structure in the VLBI maps, the factor of 4 difference in the flux density on arcsecond and milliarcsecond scales of $0722+291$ and $1512+470$ indicates that there is extended emission associated with these sources. e-MERLIN observations will thus be crucial for these sources, as they would allow us to image these sources on intermediate scales, with better sensitivity than VLBI to the diffuse emission. It is also important to investigate whether the steep spectrum is associated with the compact components or with the extended emission. Multi-frequency VLBI observations would thus be useful for spectral index analysis. The upcoming telescopes such as LOFAR and SKA will allow us to investigate these compact targets with unprecedented sensitivity and resolution presently not available at the very low frequency bands, where most of the emission in USS sources originate. Finally, from VLBI observations of this small sample we may already conclude that ultra-steep spectrum sources represent a rather diverse class of objects.

\section{References}

[1] Cohen A. S., Lane W. M., Cotton W. D., Kassim N. E., Lazio T. J., Perley R. A., Condon J. J. Erickson W. C., 2007, ApJ, 134, 1245

[2] Condon J. J., Cotton W. D., Greisen E. W., Yin Q. F., Perley R. A., Taylor G. B. Broderick J. J., 1998, Astronomical Journal, 115, 1693

[3] Becker R. H., White R. L. Helfand D. J., 1995, Astrophysical Journal, 450, 559

[4] De Breuck, C., van Breugel, W., Röttgering H.J.A. \& Miley, G., 2000, A \& A, 143, 303

[5] Krolik, J. H. \& Chen, W. 1991, AJ, 102, 1659

[6] Rengelink R. B., Tang, Y., de Bruyn A. G., et al. 1997, A \& A, 124, 259

[7] Reuland, M., Röttgering H. J. A., van Breugel, W., \& De Breuck, C. 2004, MNRAS, 353, 377

[8] Röttgering H. J. A., van Ojik R., Miley G. K., Chambers K. C., van Breugal W. J. M. de Koff S., 1997, A \& A, 326, 505

[9] Seymour, N., Stern, D., DeBreuck, C., et al. 2007, ApJ, 171, 353

[10] Van Weeren R. J., Röttgering H. J. A., Bruggen M. Cohen A., 2009, A \& A, 508, 75

[11] Venemans, B. P., Röttgering H. J. A., Miley, G. K., et al. 2007, A \& A, 461, 823 